\title{
Efficacy and Prognosis of Chemoradiotherapy for 501 Patients With Postoperative Recurrence of Esophageal Cancer
}

\section{Yan Kong}

The Fourth Hospital of Hebei Medical University

Hongmei Gao

Shijiazhaung People's Hospital

\section{Youmei Li}

The Fourth Hospital of Hebei Medical University

\section{Shuguang Li}

The Fourth Hospital of Hebei Medical University

\section{Ke Yan}

The Fourth Hospital of Hebei Medical University

Shuchai Zhu

The Fourth Hospital of Hebei Medical University

\section{WenBin Shen ( Wbshen1979@sohu.com )}

The Fourth Hospital of Hebei Medical University

\section{Research}

Keywords: Surgical treatment/recurrence, Recurrent therapy/radiotherapy, Recurrent therapy/chemotherapy, Prognosis

Posted Date: October 23rd, 2020

DOI: https://doi.org/10.21203/rs.3.rs-94545/v1

License: (c) (1) This work is licensed under a Creative Commons Attribution 4.0 International License. Read Full License 
$1 \quad$ Efficacy and prognosis of chemoradiotherapy for 501 patients with postoperative recurrence of esophageal cancer

3 Yan Kong ${ }^{1}$, Hongmei $\mathrm{Gao}^{2}$, Youmei $\mathrm{Li}^{3}$, Shuguang $\mathrm{Li}^{3}, \mathrm{Ke} \mathrm{Yan}^{3}$, Shuchai $\mathrm{Zhu}^{3}$, Wenbin 4 Shen $^{3 *}$

51 Department of Oncology, The Fourth Hospital of Hebei Medical University, 6 Shijiazhuang, Hebei 050011, China

72 Imaging Center, Shijiazhuang People’s Hospital, Shijiazhuang, Hebei 050051, China

83 Department of Radiotherapy, The Fourth Hospital of Hebei Medical University,

$9 \quad$ Shijiazhuang, Hebei 050011, China

$10{ }^{*}$ Correspondence author: WenBin Shen, Department of Radiotherapy, The Fourth

11 Hospital of Hebei Medical University, No. 12, Jiankang Road, Chang'an District,

12 Shijiazhuang, Hebei 050011, China. E-mail: Wbshen1979@sohu.com. Tel: 03111386095317.

14 Co-first author: Yan Kong ${ }^{1}$ and HongMei Gao ${ }^{2}$

15

16

17

18

19

20

21 


\section{Abstract}

23 Background: To analyze the efficacy and prognosis of chemoradiotherapy for patients

24 with recurrence of esophageal cancer.

25 Method: A single center study was conducted in 501 patients with recurrent esophageal

26 cancer who received chemotherapy and radiotherapy. Univariate or multivariate

27 analysis was performed to assess the survival, recurrence pattern, prognosis of

28 retreatment and influencing factors.

29 Result: The median time to recurrence of esophageal cancer was 11.6 months (range:

$30 \quad 0.3-87.4$ months), while the median survival time of chemoradiotherapy after

31 recurrence was 12.1 months. Multivariate analysis identified gender, $\mathrm{pN}$ stage, log odds

32 of positive lymph nodes (LODDS) value, chemotherapy cycle, recurrence time, and

33 combined distant metastasis as independent prognostic factors $(P=0.002,0.035,0.000$,

$340.000,0.000,0.001)$. In this study, 157 patients had combined combined distant

35 metastasis, and the 1-, 3-, and 5-year survival rates of these patients after radiotherapy

36 were $43.3 \%, 9.1 \%$, and $5.5 \%$, respectively. On the contrary, the $1-, 3-$, and 5-year

37 survival rates of patients with local regional recurrence (LRR) only were 53.6, 22.6\%,

38 and $16.4 \%$, respectively. Statistically, the differences in the survival rates were

39 significant between the above two groups of patients $\left(\chi^{2}=10.786, P=0.001\right)$.

40 Meanwhile, we identified recurrence time, chemotherapy cycle, and prescribed dose as

41 the significant factors affecting the prognosis among 344 patients with postoperative

42 LRR only $\left(\chi^{2}=22.605,13.957,10.446 ; P=0.000,0.000,0.005\right)$. 
43 Conclusion: This study showed that chemoradiotherapy is safe and effective in patients

44 with recurrent esophageal cancer, while male patients with late $\mathrm{pN}$ stage, high LODDS, 45 chemotherapy of $\leq 2$ cycles, recurrence time of $\leq 24$ months, or combined distant 46 metastasis had a poor prognosis.

47 Keywords: Surgical treatment/recurrence, Recurrent therapy/radiotherapy, Recurrent 48 therapy/chemotherapy, Prognosis

49

50

51

52

53

54

55

56

57

58

59

60

61

62

63 


\section{Introduction}

65 Cancer is the second leading cause of death in the world, and esophageal cancer is the

66 sixth leading cause of cancer-related death, affecting more than 450000 people

67 worldwide[1, 2]. In China, esophageal cancer is one of the common malignancies [3].

68 And there are mainly two types of esophageal cancer including squamous cell

69 carcinoma and adenocarcinoma. The main treatment methods involve RT, surgery and

70 chemotherapy. Surgical treatment is the main treatment mode for patients with

71 esophageal cancer who can be surgically resected, but the clinical efficacy of radical

72 resection remains unsatisfactory $[4,5]$. The treatment failure can be mainly attributed to

73 local regional recurrence (LRR) and combined distant metastasis in which LRR takes

74 up $30 \%$ to $40 \%$ of the cases with 5 -year survival rarely exceeding $25 \%[6,7]$. Once the

75 treatment failure occurs, most patients have a poor prognosis and die within 1 year[8,

76 9]. At present, the main salvage treatment options for patients with postoperative

77 recurrence include surgery, radiotherapy, chemotherapy, and chemoradiotherapy, while

78 the curative effect is not ideal[10-12], and the optimal treatment regimen needs to be

79 determined. Neoadjuvant radiotherapy and chemotherapy can improve the overall

80 survival rate (OS) and local tumor control rate of patients, but neoadjuvant radiotherapy

81 often increases the difficulties associated with surgery due to tissue edema and

82 hemorrhage. Moreover, the patients in China usually choose surgery rather than

83 chemoradiotherapy as their initial treatment option[13].

84 In addition, no consensus on radiotherapy target area, radiotherapy dose, and 
combined chemotherapy regimens for patients with postoperative recurrence leads to unsatisfactory outcomes of chemoradiotherapy $[12,14]$. In this single center study, we aimed to further clarify the efficacy of chemoradiotherapy for patients with recurrent esophageal cancer and identify relevant prognostic factors. (1)

\section{Materials and Methods}

A single-center study of 501 esophageal cancer patients was conducted. All patients with a male to female ratio of 2.7:1 were admitted to the Fourth Hospital of Hebei Medical University from January 2009 to December 2014. This study has been approved by the Ethics Committee of Fourth Hospital of Hebei Medical University (2020KY382) and all subjects participated in the study with a written informed consent.

\section{Inclusion and exclusion criteria}

The following inclusion criteria were used: (1) patients who received radical surgical treatment for thoracic esophageal cancer, but did not have a history of neoadjuvant treatment prior to the surgery; (2) definitely diagnosed as squamous cell carcinoma and the absence of combined distant metastasis, as evidenced by postoperative pathological examination; (3) patients who have not received adjuvant chemoradiation or chemotherapy after surgery; (4) patients at first recurrence; (5) patients with karnofsky score of $\geq 70$; (6) complicated by multiple local/regional failures and/or combined distant metastasis; (7) those who have no serious underlying diseases that affect 
treatment and can tolerate chemoradiotherapy; and (8) no history of other complicated

107 malignancies.

108 The exclusion criteria were as follows: (1) patients who received preoperative

109 neoadjuvant therapy or postoperative adjuvant therapy; (2) diagnosed as non-squamous

110 cell carcinoma evidenced by postoperative pathology; (3) those who suffer from

111 underlying diseases that seriously affect treatment and cannot receive

112 chemoradiotherapy; (4) complicated by other malignancies; and (5) those who are

113 reluctant to receive adjuvant therapy or do not receive the therapy due to the doctor's

114 recommendations.

115

116 Clinical and pathological data

117 The median age at surgery was 59 years (range: $38-78$ years), while the median age at

118 chemoradiotherapy for postoperative recurrence was 60.1 years (range: 38.8-81.9

119 years). Among all patients, 115, 24, and 25 had a history of hypertension, diabetes, and

120 coronary heart diseases, respectively. Notably, out of the above 164 cases, while 3

121 suffered from all the three types of diseases, 19 had any two of them. The median

122 esophageal length shown in barium meal angiography before operation was $5.0 \mathrm{~cm}$

123 (range: $1.0-15.0 \mathrm{~cm})$. Among all 501 patients, $431(86.0 \%)$ underwent left thoracotomy,

124 while $26(5.2 \%), 14(2.8 \%)$, and $30(6.0 \%)$ received right thoracotomy, cervical and

125 abdominal incisions, and three incisions of neck, chest and abdomen, respectively.

126 Based on degree of postoperative tissue differentiation, the patients were classified as 
127 undifferentiated (40 cases), poorly differentiated (67 cases), moderately differentiated

128 ( 7 cases) and well differentiated (386 cases). Postoperative pathological examination

129 identified 37 cases with positive upper edge of the stump and 3 cases with positive

130 lower edge, while showing no case with any nerve invasion (Table 1).

\section{Log odds of metastatic lymph nodes}

133 Log odds of metastatic lymph nodes (LODDS) were calculated as follows:

134 LODDS=Log (number of positive lymph nodes +0.5 )/ (number of negative lymph

135 nodes +0.5$)$

136

137 Local/regional recurrence after treatment

138 Local/regional recurrence after treatment mainly involves anastomoses and regional

139 lymph nodes. Anastomotic recurrence needs to be confirmed by electronic gastroscopy

140 bite pathological examination. For diagnosis of lymph node metastasis, needle biopsy

141 pathology was performed to confirm metastatic superficial lymph nodes, while CT,

142 MRI, PET/CT and/or B-ultrasound were conducted to diagnose lymph node metastasis

143 in the remaining areas. Regional lymph node metastasis involves lymph nodes located

144 in the supraclavicular area, mediastinum and abdominal cavity.

146 Chemoradiotherapy

147 All 501 patients received postoperative radiotherapy; among them, 362 patients 
148

149

150

151

152

154 chemoradiotherapy. After radiotherapy, some patients continued to receive

155 consolidation chemotherapy. In this study, the median chemotherapy cycle was 3 (range:

156 1-7). And 168 patients received chemotherapy of $\geq 3$ cycles.

\section{Follow up}

159 The follow-up mainly involved telephone follow-up and outpatient review, combined

160 with a review of case data. During the period from the operation date to December 31 ,

1612019 , the patients were reviewed every 3-6 months in the first year, and then every 6-

16212 months thereafter. Of 501 patients, $36(7.2 \%)$ were lost during the follow-up. We

163 counted the patients who were lost to follow-up as death cases on the date of the last

164 follow-up.

165

\section{Statistical Analysis}

167 SPSS 19.0 software was used to perform statistical analysis. Univariate survival 168 analysis and multivariate analysis were carried out by using Log-rank test and Kaplan- 

Meier method, and Cox proportional hazards model, respectively. $P<0.05$ indicates that

170 the difference is statistically significant.

172 Results

\section{Postoperative recurrence}

174 The median postoperative recurrence time for all patients was 11.6 months (range: 0.3-

17587.4 months). The recurrence occurred in the following sites: 84 cases (16.8\%) with

176 simple supraclavicular lymph node recurrence, 254 cases (50.7\%) with simple

177 mediastinal lymph node recurrence, 27 cases (5.4\%) with simple anastomotic

178 recurrence, 28 cases $(5.6 \%)$ with simple abdominal lymph node recurrence, 52 cases

$179(10.4 \%)$ with of the recurrence of supraclavicular and mediastinal lymph nodes, 10

180 cases $(2.0 \%)$ with the recurrence of supraclavicular and abdominal lymph nodes, 4

181 cases $(0.8 \%)$ with the recurrence of supraclavicular lymph nodes and anastomoses, 23

182 cases (4.6\%) with the recurrence of mediastinal lymph nodes and anastomoses, 11 cases

$183(2.2 \%)$ with the recurrence of mediastinum and abdominal lymph nodes, 2 cases $(0.4 \%)$

184 with the recurrence of anastomoses and abdominal lymph nodes, 2 cases $(0.4 \%)$ with

185 the recurrence of the lock, mediastinal and abdominal lymph nodes, and 4 cases $(0.8 \%)$

186 with the recurrence of the lock and mediastinal lymph nodes, and anastomoses.

187 We further reported 157 cases with distant metastases among all the patients. The

188 clinical study showed that out of 157 cases, 8 developed combined distant metastasis

189 earlier than recurrence, while combined distant metastasis and recurrence were detected 

metastasis was 5.4-94.4 months, and the median recurrence time was 27.3 months.

\section{Survival outcome}

194 The 1-, 3-, and 5-year survival rates of all postoperative patients were $88.8 \%, 43.8 \%$, and $27.3 \%$, respectively, while the median survival time was 31.0 months. The 1-, 3-, and 5-year survival rates of patients with radiotherapy following postoperative recurrence were $50.3 \%, 18.1 \%$, and $12.8 \%$, respectively, and the median survival time was 12.1 months.

200 prognosis: gender $\left(\chi^{2}=11.427, P=0.001\right)$, smoking $\left(\chi^{2}=7.385, P=0.007\right)$, drinking $201\left(\chi^{2}=8.079, P=0.004\right)$, length of lesion in esophagography $\left(\chi^{2}=7.140, P=0.008\right), \mathrm{pT}$ 202 stage $\left(\chi^{2}=9.607, P=0.022\right)$, pN stage $\left(\chi^{2}=10.214, P=0.006\right)$, vascular tumor thrombus $203\left(\chi^{2}=4.232, P=0.040\right)$, LODDS $\left(\chi^{2}=25.233, P=0.000\right)$, chemotherapy cycles $204\left(\chi^{2}=19.937, P=0.000\right)$, radiation dose $\left(\chi^{2}=8.417, P=0.015\right)$, recurrence time $205\left(\chi^{2}=25.616, P=0.000\right)$, and combined distant metastasis $\left(\chi^{2}=10.786, P=0.001\right)$.

206 Conversely, there were no significant correlations between the prognosis and various 207 factors including age, underlying diseases, family history, location of the lesions, 208 method of thoracotomy, postoperative stump, number of dissected lymph nodes, 209 postoperative chemotherapy, irradiation method, and number of recurring areas (Table $2101)$. 
212 all patients such as gender $(P=0.002)$, pN stage $(P=0.035)$, LODDS $(P=0.000)$,

213 chemotherapy cycles $(P=0.000)$, recurrence time $(P=0.000)$, and combined distant

214 metastasis $(P=0.001)$ (Table 2$)$.

216 Table 2 Multivariate analysis of prognosis of patients with esophageal cancer

217 recurrence after radiotherapy $(\boldsymbol{n}=\mathbf{5 0 1})$

\begin{tabular}{ccccccc}
\hline Prognostic factors & B & SE & Wald & P & OB & $\mathbf{9 5 \%} \boldsymbol{C I}$ \\
\hline Sex & -0.374 & 0.122 & 9.408 & 0.002 & 0.688 & $0.542-0.874$ \\
pN stage & -0.222 & 0.106 & 4.435 & 0.035 & 0.801 & $0.651-0.985$ \\
LODDS & 0.507 & 0.105 & 23.292 & 0.000 & 1.661 & $1.352-2.041$ \\
Chemotherapy cycle & -0.488 & 0.113 & 18.587 & 0.000 & 0.614 & $0.492-0.766$ \\
$\begin{array}{c}\text { Postoperative } \\
\text { recurrence time }\end{array}$ & -0.601 & 0.140 & 18.368 & 0.000 & 0.548 & $0.417-0.722$ \\
$\begin{array}{c}\text { Combined distant } \\
\text { metastasis }\end{array}$ & 0.355 & 0.109 & 10.571 & 0.001 & 1.427 & $1.152-1.767$ \\
\hline
\end{tabular}

220 As shown in Fig. 1, a total of 157 patients developed combined combined distant

221 metastasis. The 1-, 3-, and 5-year survival rates of patients with combined distant

222 metastasis after radiotherapy were $43.3 \%, 9.1 \%$, and $5.5 \%$, respectively, and the median

223 survival time was 11.1 months. On the contrary, the 1-, 3-, and 5-year survival rates of

224 patients with LRR only after radiotherapy were 53.6, $22.6 \%$, and $16.4 \%$, respectively,

225 and the median survival time was 12.8 months. Statistically, the above differences were

226 significant $\left(\chi^{2}=10.786, P=0.001\right)$. 
229 A total of 344 patients had local recurrence. As shown in Table 3 and Fig. 2, the

230 subgroup univariate analysis identified postoperative recurrence time $\left(\chi^{2}=22.605\right.$,

$231 P=0.000)$, chemotherapy cycles $\left(\chi^{2}=13.957, P=0.000\right)$, and prescription dose

232 ( $\left.\chi^{2}=10.446, P=0.005\right)$ as the significant factors affecting the prognosis.

\section{Discussion}

235 At present, there are no standard treatment regimens for esophageal cancer recurrence.

236 The 2016 guidelines issued by Union for International Cancer Control (UICC)

237 recommended that the recurrence after surgery should be treated with concurrent

238 chemoradiotherapy, while combined chemotherapy based on fluorouracil or taxanes

239 can be used as the preferred concurrent chemoradiotherapy. Moreover, patients with the

240 recurrence after surgery may receive surgery, chemotherapy, and palliative support

241 treatment. Clearly, the above recommendation was made based on the non-surgical

242 treatment mode of esophageal cancer, a retrospective analysis of a small sample size,

243 and indirect evidence from other studies. Previous reports have shown that

244 chemoradiotherapy can benefit the survival of some patients with recurrence of

245 esophageal cancer after surgery. However, the following issues remain to be addressed

246 for each research center: the dose of radiotherapy, the range of irradiation target area,

247 the necessity of combined radiotherapy with chemotherapy, and the mode of combined

248 treatment $[12,14,15]$. So far, there have been numerous studies on chemoradiotherapy 
249 administered to patients with recurrent esophageal cancer [16-21]. Among these studies, 250 the survival rates of patients varied greatly from one to another. In these cases, the 251 median survival time was 7-43 months. 1-, 2-, and 3-year survival rates were 33.8\% $25285.7 \%, 15.0 \%-51.3 \%$, and $10.6 \%-56.3 \%$, respectively, while there were few reports 253 on 5-year and over five years survival rates. The above observations could be explained 254 as follows: (1) most of the studies were retrospective analysis with a small sample size; 255 (2) there were inherent biases in case selection; (3) patients either with local recurrence 256 only or with combined local recurrence and combined distant metastasis were 257 differentially included in different studies; (4) the dose and strategies of radiotherapy 258 or chemotherapy regimens varied from one study to another; (5) patients with 259 concurrent chemoradiotherapy were included in a subset of studies, while other studies 260 only involved patients with radiotherapy, and no subgroup analysis was performed.

261 Given the number of cases involved (501 patients), the present study may be the largest 262 retrospective study on chemoradiotherapy administered to patients with recurrent 263 esophageal cancer in a single research center. This study showed that the 1-, 3-, and 5264 year survival rates were $50.3 \%, 18.1 \%$ and $12.8 \%$, respectively, while the median 265 survival time was 12.1 months. Among the total of 501 cases with local recurrence, 157 266 developed combined distant metastasis. For 344 patients with local recurrence only, the 2671 -, 3-, and 5-year survival rates were $53.6 \%, 22.6 \%$, and $16.4 \%$, respectively, and the 268 median survival time was 12.8 months.

269 Previous studies have identified various prognostic factors for patients with 
$27122-25]$. Among these factors, the time interval between surgery and the occurrence of

272 recurrence has been confirmed by most studies to be an independent factor affecting

273 the prognosis. Jingu et al.[24] retrospectively studied the clinical data of 80 patients

274 with postoperative local recurrence of esophageal cancer by a multivariate analysis, and

275 found that the short postoperative recurrence time is one of the independent factors

276 affecting the overall survival of patients. Nemoto et al.[25] reported similar results in

277 the retreatment of 33 patients with postoperative local recurrence of esophageal cancer,

278 and identified the postoperative recurrence interval as one of the independent factors

279 affecting the prognosis of patients. Consistently, the multivariate analysis in our study

280 demonstrated that the interval between surgery and recurrence is among the

281 independent factors affecting the prognosis. The analysis of 344 patients with

282 postoperative local recurrence only further confirmed that the interval significantly

283 affects the prognosis of patients. It has been shown that the occurrence of recurrence or

284 metastasis in patients with esophageal cancer shortly after surgery may be related to the

285 micro-metastasis of tumor cells[26]. It is known that esophageal cancer is a systemic

286 disease, rather a localized one. As a local treatment, radical surgery has no effect on the

287 micro-metastasis that occurs prior to the operation. Patients with early relapse may have

288 a poorer biological behavior than those with later relapse, showing a prognostic

289 difference between the two groups of patients. The TNM staging of esophageal cancer

290 acts as an indicator of tumor biological behavior in the patients, and is closely correlated 
291 with postoperative recurrence and prognosis of patients with esophageal cancer. Similar

292 to the report of Hsu et al.[27], the present study showed that postoperative $\mathrm{N}$ staging is

293 an independent factor affecting the prognosis of patients. The prognosis of patients with

294 postoperative recurrence and combined distant metastasis is usually poorer than that of

295 patients with recurrence only. Generally, the recurrence of esophageal cancer mainly

296 involves lymph nodes and anastomoses. Notably, clinical symptoms and signs related

297 to the recurrence, such as neck mass, hoarseness, cough, and poor swallowing appear

298 earlier, whereas other recurrence-caused clinical symptoms and signs involving lung,

299 liver, bone, brain, and other organs occur relatively late. The cancer patients detected at

300 an early stage can be diagnosed and treated in a timely manner, thereby affecting the

301 prognosis of patients. As a localized treatment regimen, radiotherapy displays a limited

302 efficacy for patients with distant metastases. And patients with esophageal cancer are

303 less sensitive to chemotherapy drugs, suggesting a limited effect of chemotherapy on

304 the patients $[11,28]$. Thus, patients with combined recurrence and combined distant

305 metastasis usually have a poor prognosis. Most clinical studies have demonstrated that

306 combined chemoradiotherapy is superior to radiotherapy or chemotherapy alone,

307 providing an important treatment regimen for patients with postoperative recurrence of

308 esophageal cancer $[6,8,12,14-17]$.

309 Here, we showed that the combined chemotherapy did not affect the prognosis of

310 the whole group of patients. However, further stratified analysis revealed that

311 chemotherapy of more than two cycles led to a better prognosis in the patients, while 
312 independently affecting the prognosis. It has been rarely studied whether the number of

313 chemotherapy cycles has an impact on the prognosis of patients with relapsed

314 esophageal cancer treated with chemoradiotherapy. And fewer studies on the subgroup

315 analysis related to the cycle number of chemotherapies have been reported. Among the

316 related studies, synchronous chemotherapy of two cycles was normally described in the

317 materials and methods [22]. Clearly, most studies were aimed at postoperative adjuvant

318 chemotherapy[29-31], while the cycle number of chemotherapies varied from one study

319 to another. Rice et al.[29] and Heroor et al.[30] recommended a regimen involving

320 postoperative radiotherapy and concurrent chemotherapy of 2 cycles. Meanwhile,

321 Bedard et al.[31] showed that patients undergoing postoperative radiotherapy combined

322 with chemotherapy of 4 cycles have a better prognosis. Thus, to determine the optimal

323 cycle number of chemotherapies for patients with postoperative recurrence of

324 esophageal cancer, prospective studies of large samples need to be conducted. Besides,

325 the dose of radiotherapy for patients with postoperative recurrence of esophageal cancer

326 has been an interesting issue for the physicians. Since the dose of radiotherapy is

327 generally affected by a number of factors such as the general health status of patients

328 during treatment, the location of the recurring lesion, administration of combined

329 chemotherapy, and the size and scope of the lesion, there are differences in the dose of

330 radiotherapy among the studies. At present, while there is no consensus on the radiation

331 dose, most studies recommend that the radiation should be conducted at a dose of no

332 less than $50 \mathrm{~Gy}$. Zhang et al.[32] found that the median overall survival and progression- 
333 free survival of patients with postoperative recurrence of esophageal cancer receiving

334 radiotherapy at a dose of $\geq 60$ Gy were higher than those of patients undergoing 335 radiotherapy at a dose of $<60$ Gy. Thus, they recommended that the radiation dose for 336 patients with regional recurrence of esophageal cancer should be $\geq 60 \mathrm{~Gy}$. Ni et al.[33] 337 analyzed 193 cases with postoperative recurrence of thoracic esophageal squamous cell 338 carcinoma undergoing re-therapy and observed that the prognosis of patients in high339 dose radiation group ( $\geq 60 \mathrm{~Gy}$ ) was better than that in lower dose group. Shioyama et 340 al.[34] studied 82 patients with local recurrence of esophageal cancer after surgery and 341 showed that the prognosis of patients receiving $\geq 50$ Gy irradiation was better than that 342 in lower dose group. Similar to the above findings, the present study demonstrated that 343 the patients with a radiotherapy at a dose of less than 60 Gy had a poor prognosis.

\section{Conclusion}

346 This study showed that chemoradiotherapy is safe and effective for patients with 347 postoperative recurrence of esophageal cancer. Male patients with late $\mathrm{pN}$ stage, higher 348 LODDS, chemotherapy of $\leq 2$ cycles, recurrence time of $\leq 24$ months, and combined 349 distant metastasis displayed a poor prognosis. Since the present study is a single-center 350 retrospective study, certain biases in case collection, number of cases between groups, 351 and treatment methods may affect the results of this study. Moreover, this study may be 352 subject to the potential influencing factors such as ECOG score, KPS score, regression 353 status, age, size and location of the recurrent lesion. Thus, a multi-center randomized 
354 controlled study in a large cohort needs to be conducted to provide a more reliable basis

355 for the clinical treatment of patients with postoperative recurrence of esophageal cancer.

\section{Abbreviations}

358 LRR: Local regional recurrence; OS: Overall survival rate; LODDS: Log odds of 359 metastatic lymph nodes; ENI: Elective nodal irradiation; IFI: Involved-field irradiation;

360 UICC: Union for International Cancer Control

\section{Acknowledgements}

363 Not applicable

\section{Authors' contributions}

366 WS, and YL drafted the manuscript. YK and HG participated in the design of the study

367 and performed the statistical analysis. SL, and KY conceived the study and participated 368 in its design and coordination. SZ revised the manuscript. All authors read and approved 369 the final manuscript.

371 Funding

372 The present study did not receive any funding. 
378 Ethical consent was obtained from the Ethics Committee of Fourth Hospital of Hebei

379 Medical University (2020KY382) and all subjects participated in the study with a 380 written informed consent.

\section{Consent for publication}

Not applicable

\section{Conflict of interest}

386 The authors declare that they have no competing interests.

\section{References}

389 1. Global Burden of Disease Cancer C, Fitzmaurice C, Dicker D, Pain A, Hamavid H, Moradi-Lakeh M, et al. The Global Burden of Cancer 2013. JAMA Oncol. $2015 ; 1: 505-27$

392 2. Global Burden of Disease Cancer C, Fitzmaurice C, Allen C, Barber RM, Barregard L, Bhutta ZA, et al. Global, Regional, and National Cancer Incidence, Mortality, Years of Life Lost, Years Lived With Disability, and Disability- 
397 3. Chen W, Zheng R, Baade PD, Zhang S, Zeng H, Bray F, et al. Cancer statistics in China, 2015. CA Cancer J Clin. 2016;66:115-32.

399 4. Rice TW, Ishwaran H, Ferguson MK, Blackstone EH, Goldstraw P. Cancer of 400 the Esophagus and Esophagogastric Junction: An Eighth Edition Staging Primer. J Thorac Oncol. 2017;12:36-42.

5. Tanaka T, Matono S, Nagano T, Shirouzu K, Fujita H, Yamana H. Esophagectomy with extended lymphadenectomy for submucosal esophageal cancer: long-term outcomes and prognostic factors. Ann Surg Oncol. 2012;19:750-6.

6. Oppedijk V, van der Gaast A, van Lanschot JJ, van Hagen P, van Os R, van Rij 2014;32:385-91.

410 7. Guo XF, Mao T, Gu ZT, Ji CY, Fang WT, Chen WH. Clinical study on 411 postoperative recurrence in patients with pN0 esophageal squamous cell 412 carcinoma. J Cardiothorac Surg. 2014;9:150.

413 8. Shimada H, Kitabayashi H, Nabeya Y, Okazumi S, Matsubara H, Funami Y, et 414 al. Treatment response and prognosis of patients after recurrence of esophageal $415 \quad$ cancer. Surgery. 2003;133:24-31.

416 9. Ohtsu A. Chemoradiotherapy for esophageal cancer: current status and 
perspectives. Int J Clin Oncol. 2004;9:444-50.

418 10. Lin D, Jiang S, Ma L, Hu H, Ye T, Shao L, et al. Clinical analyses on salvage lymphadenectomy through cervical incision for patients with cervical and cervicothoracic recurrences after esophagectomy. J Thorac Dis. 2017;9:3832-9.

421 11. Kosuga T, Shiozaki A, Fujiwara H, Ichikawa D, Okamoto K, Komatsu S, et al. Treatment outcome and prognosis of patients with lymph node recurrence of thoracic esophageal squamous cell carcinoma after curative resection. World J Surg. 2011;35:798-804.

12. Kawamoto T, Nihei K, Sasai K, Karasawa K. Clinical outcomes and prognostic factors of chemoradiotherapy for postoperative lymph node recurrence of esophageal cancer. Jpn J Clin Oncol. 2018;48:259-64.

13. Liu Q, Cai XW, Wu B, Zhu ZF, Chen HQ, Fu XL. Patterns of failure after radical surgery among patients with thoracic esophageal squamous cell carcinoma: implications for the clinical target volume design of postoperative radiotherapy.

432 14. Zhang WW, Zhu YJ, Yang H, Wang QX, Wang XH, Xiao WW, et al. Concurrent radiotherapy and weekly chemotherapy of 5-fluorouracil and platinum agents radical resection of esophageal squamous cell carcinoma. Radiat Oncol. 
439 16. Bao Y, Liu S, Zhou Q, Cai P, Anfossi S, Li Q, et al. Three-dimensional conformal radiotherapy with concurrent chemotherapy for postoperative recurrence of esophageal squamous cell carcinoma: clinical efficacy and failure pattern. Radiat Oncol. 2013;8:241.

443 17. Kobayashi R, Yamashita H, Okuma K, Shiraishi K, Ohtomo K, Nakagawa K. Salvage radiation therapy and chemoradiation therapy for postoperative locoregional recurrence of esophageal cancer. Dis Esophagus. 2014;27:72-8.

446 18. Ma X, Zhao K, Guo W, Yang S, Zhu X, Xiang J, et al. Salvage squamous cell carcinoma. Ann Surg Oncol. 2015;22:624-9.

19. Su XD, Zhang DK, Zhang X, Lin P, Long H, Rong TH. Prognostic factors in patients with recurrence after complete resection of esophageal squamous cell

20. Raoul JL, Le Prise E, Meunier B, Julienne V, Etienne PL, Gosselin M, et al. Combined radiochemotherapy for postoperative recurrence of oesophageal cancer. Gut. 1995;37:174-6.

21. Jingu K, Nemoto K, Matsushita H, Takahashi C, Ogawa Y, Sugawara T, et al. Results of radiation therapy combined with nedaplatin (cis-diammineglycoplatinum) and 5-fluorouracil for postoperative locoregional recurrent 
esophageal cancer. BMC Cancer. 2006;6:50.

460 22. Jingu K, Matsushita H, Takeda K, Umezawa R, Takahashi C, Sugawara T, et al. Long-term results of radiotherapy combined with nedaplatin and 5-fluorouracil for postoperative loco-regional recurrent esophageal cancer: update on a phase II study. BMC Cancer. 2012;12:542.

23. Kimoto T, Yamazaki H, Suzuki G, Aibe N, Masui K, Tatekawa K, et al. Local field radiotherapy without elective nodal irradiation for postoperative locoregional recurrence of esophageal cancer. Jpn J Clin Oncol. 2017;47:809-14.

24. Jingu K, Umezawa R, Yamamoto T, Matsushita H, Ishikawa Y, Kozumi M, et al. Elective nodal irradiation is not necessary in chemoradiotherapy for postoperative loco-regional recurrent esophageal cancer. Jpn J Clin Oncol.

25. Nemoto K, Ariga H, Kakuto Y, Matsushita H, Takeda K, Takahashi C, et al. Radiation therapy for loco-regionally recurrent esophageal cancer after surgery. Radiother Oncol. 2001;61:165-8.

26. Raj GV, Moreno JG, Gomella LG. Utilization of polymerase chain reaction technology in the detection of solid tumors. Cancer. 1998;82:1419-42.

27. Hsu PK, Wang BY, Huang CS, Wu YC, Hsu WH. Prognostic factors for postrecurrence survival in esophageal squamous cell carcinoma patients with recurrence after resection. J Gastrointest Surg. 2011;15:558-65.

479 28. Nakamura T, Ota M, Narumiya K, Sato T, Ohki T, Yamamoto M, et al. 

curative resection. Ann Surg Oncol. 2008;15:2451-7.

29. Bedard EL, Inculet RI, Malthaner RA, Brecevic E, Vincent M, Dar R. The role of surgery and postoperative chemoradiation therapy in patients with lymph node positive esophageal carcinoma. Cancer. 2001;91:2423-30.

30. Heroor A, Fujita H, Sueyoshi S, Tanaka T, Toh U, Mine T, et al. Adjuvant 486 chemotherapy after radical resection of squamous cell carcinoma in the thoracic esophagus: who benefits? A retrospective study. Dig Surg. 2003;20:229-235; discussion 236-27.

31. Rice TW, Adelstein DJ, Chidel MA, Rybicki LA, DeCamp MM, Murthy SC, et al. Benefit of postoperative adjuvant chemoradiotherapy in locoregionally advanced esophageal carcinoma. J Thorac Cardiovasc Surg. 2003;126:1590-6.

32. Zhang J, Peng F, Li N, Liu Y, Xu Y, Zhou L, et al. Salvage concurrent radiochemotherapy for post-operative local recurrence of squamous-cell esophageal cancer. Radiat Oncol. 2012;7:93.

33. Ni W, Yang J, Deng W, Xiao Z, Zhou Z, Zhang H, et al. Patterns of recurrence after surgery and efficacy of salvage therapy after recurrence in patients with thoracic esophageal squamous cell carcinoma. BMC Cancer. 2020;20:144.

498 34. Shioyama Y, Nakamura K, Ohga S, Nomoto S, Sasaki T, Yamaguchi T, et al. 499 Radiation therapy for recurrent esophageal cancer after surgery: clinical results and prognostic factors. Jpn J Clin Oncol. 2007;37:918-23. 
502 Fig. 1: Survival curves of the cancer patients with or without combined combined 503 distant metastasis.

504 Fig. 2: Single factor subgroup analysis curve of patients with postoperative local 505 recurrence only. A: recurrence time, B: chemotherapy cycles, C: prescription dose. 506 507 508 509 510 511 512 513 
522 Table 1 Univariate analysis of prognosis in patients with recurrent esophageal

523 cancer after radiotherapy $(\boldsymbol{n}=\mathbf{5 0 1})$

\begin{tabular}{|c|c|c|c|c|c|c|c|c|}
\hline \multicolumn{2}{|c|}{ Prognostic factors } & \multirow[t]{2}{*}{$\mathbf{N}$} & \multicolumn{3}{|c|}{ Survival rate $(\%)$} & \multirow{2}{*}{$\begin{array}{c}\text { Median survival rate } \\
\text { (months) }\end{array}$} & \multirow[t]{2}{*}{$\chi^{2}$} & \multirow[t]{2}{*}{$P$} \\
\hline & & & $1-y r$ & $3-y r$ & $5-y r$ & & & \\
\hline \multirow[t]{3}{*}{ Sex } & & & & & & & 11.427 & 0.001 \\
\hline & Male & 366 & 45.3 & 14.8 & 9.2 & 11.1 & & \\
\hline & Female & 135 & 63.8 & 26.9 & 22.1 & 16.2 & & \\
\hline \multirow[t]{3}{*}{ Age } & & & & & & & 2.38 & 0.123 \\
\hline & $\leq 59 \mathrm{y}$ & 265 & 52.4 & 19.4 & 16.5 & 12.6 & & \\
\hline & $\geq 60 \mathrm{y}$ & 236 & 48.0 & 16.7 & 8.4 & 11.7 & & \\
\hline \multirow[t]{3}{*}{ Basic diseases } & & & & & & & 0.172 & 0.679 \\
\hline & No & 362 & 47.2 & 17.8 & 13.9 & 11.6 & & \\
\hline & Yes & 139 & 58.6 & 19.2 & 10.3 & 13.0 & & \\
\hline \multirow[t]{3}{*}{ Smoking } & & & & & & & 7.385 & 0.007 \\
\hline & No & 238 & 57.6 & 23.0 & 15.8 & 14.6 & & \\
\hline & Yes & 263 & 43.5 & 13.2 & 10.1 & 10.6 & & \\
\hline \multirow[t]{3}{*}{ Drinking } & & & & & & & 8.079 & 0.004 \\
\hline & No & 295 & 54.3 & 23.1 & 16.0 & 13.9 & & \\
\hline & Yes & 206 & 44.2 & 10.6 & 7.9 & 11.1 & & \\
\hline \multirow[t]{3}{*}{ Family } & & & & & & & 1.861 & 0.173 \\
\hline & No & 429 & 49.6 & 20.1 & 14.4 & 11.9 & & \\
\hline & Yes & 72 & 54.7 & 4.8 & 4.8 & 12.5 & & \\
\hline \multirow[t]{3}{*}{ Differentiation } & & & & & & & 0.020 & 0.887 \\
\hline & No/poorly differentiated & 107 & 46.4 & 20.4 & 15.3 & 11.3 & & \\
\hline & Medium/highly differentiated & 394 & 51.3 & 17.6 & 12.3 & 12.3 & & \\
\hline \multirow[t]{4}{*}{ Lesion location } & & & & & & & 4.640 & 0.098 \\
\hline & Upper thoracic & 60 & 46.9 & 24.1 & 18.7 & 11.0 & & \\
\hline & Middle thoracic & 275 & 52.0 & 21.7 & 14.8 & 12.7 & & \\
\hline & Lower thoracic & 166 & 48.5 & 8.8 & 7.3 & 11.8 & & \\
\hline \multirow[t]{3}{*}{ Thoracotomy } & & & & & & & 0.011 & 0.917 \\
\hline & Left thoracotomy & 431 & 49.3 & 18.3 & 12.9 & 11.8 & & \\
\hline & other & 70 & 56.7 & 17.0 & 12.1 & 13.9 & & \\
\hline \multirow{3}{*}{$\begin{array}{l}\text { Length of lesion in } \\
\text { esophagography (cm) }\end{array}$} & & & & & & & 7.140 & 0.008 \\
\hline & $<5.0$ & 218 & 54.8 & 22.7 & 18.2 & 14.2 & & \\
\hline & $\geq 5.0$ & 283 & 46.7 & 14.9 & 9.4 & 11.3 & & \\
\hline \multirow[t]{4}{*}{ pT stage } & & & & & & & 9.607 & 0.022 \\
\hline & pT1 & 66 & 50.5 & 18.6 & 16.2 & 12.1 & & \\
\hline & pT2 & 100 & 61.7 & 27.1 & 20.9 & 16.1 & & \\
\hline & pT3 & 314 & 47.5 & 15.5 & 10.4 & 11.6 & & \\
\hline
\end{tabular}




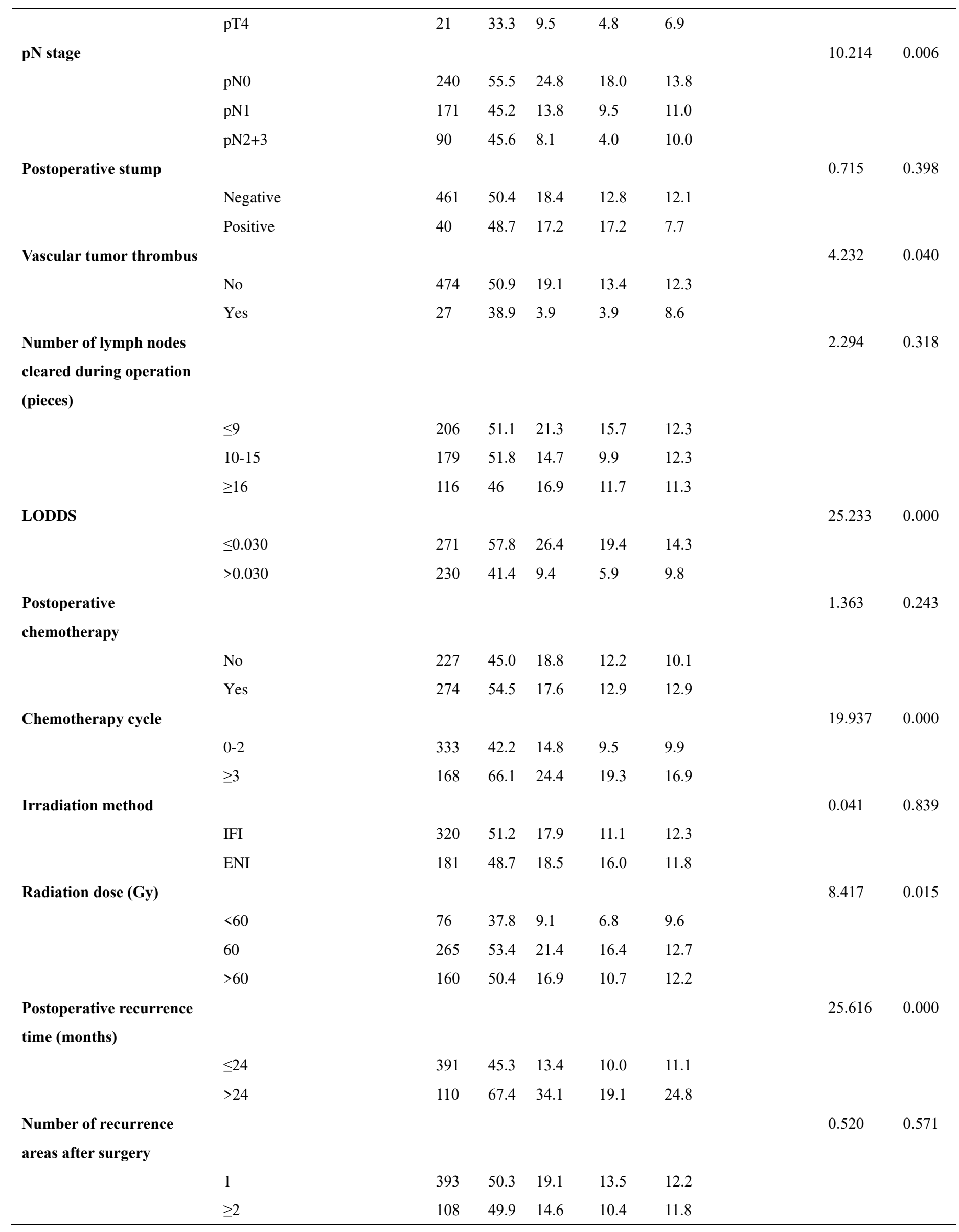


metastasis

$\begin{array}{llllll}\text { No } & 344 & 53.6 & 22.6 & 16.4 & 12.8 \\ \text { Yes } & 157 & 43.4 & 9.1 & 5.5 & 11.1\end{array}$

524 LODDS: log odds of positive lymph nodes

525

526

527

528

529

530

531

532

533

534

535

536

537

538

539

540

541

542

543

544

545

546

547

548

549

550

551

552

553

554 
Table 3 Univariate subgroup analysis results of patients with only local regional

556 recurrence after surgery

\begin{tabular}{|c|c|c|c|c|c|c|c|c|}
\hline \multirow[t]{2}{*}{ Prognostic factors } & & \multirow[t]{2}{*}{$\mathbf{N}$} & \multicolumn{3}{|c|}{ Survival rate $(\%)$} & \multirow{2}{*}{$\begin{array}{l}\text { Median survival } \\
\text { rate (months) }\end{array}$} & \multirow[t]{2}{*}{$\chi^{2}$} & \multirow[t]{2}{*}{$\boldsymbol{P}$} \\
\hline & & & $1-y r$ & $3-y r$ & $5-y r$ & & & \\
\hline \multirow{4}{*}{$\begin{array}{l}\text { Postoperative recurrence time } \\
\text { (months) }\end{array}$} & & & & & & & 22.605 & 0.000 \\
\hline & & & & & & & & \\
\hline & $\leq 24$ & 262 & 48.2 & 16.0 & 12.0 & 11.6 & & \\
\hline & $>24$ & 82 & 70.1 & 42.5 & 26.6 & 28.9 & & \\
\hline \multirow{4}{*}{$\begin{array}{c}\text { Number of recurrence areas after } \\
\text { surgery }\end{array}$} & & & & & & & 0.784 & 0.376 \\
\hline & & & & & & & & \\
\hline & 1 & 274 & 55.6 & 30.6 & 17.0 & 13.4 & & \\
\hline & $\geq 2$ & 70 & 44.7 & 18.6 & 13.9 & 10.9 & & \\
\hline \multirow[t]{3}{*}{ Postoperative chemotherapy } & & & & & & & 0.012 & 0.912 \\
\hline & No & 156 & 50.2 & 25.8 & 16.2 & 12.2 & & \\
\hline & Yes & 188 & 56.3 & 20.1 & 15.7 & 13.9 & & \\
\hline \multirow[t]{3}{*}{ Chemotherapy cycle } & & & & & & & 13.957 & 0.000 \\
\hline & $0-2$ & 232 & 45.8 & 17.9 & 11.6 & 10.6 & & \\
\hline & $\geq 3$ & 112 & 68.6 & 32.1 & 26.7 & 19.4 & & \\
\hline \multirow[t]{3}{*}{ Irradiation method } & & & & & & & 0.851 & 0.356 \\
\hline & IFI & 214 & 53.4 & 21.5 & 13.5 & 12.5 & & \\
\hline & ENI & 130 & 53.9 & 24.3 & 20.8 & 13.9 & & \\
\hline \multirow[t]{4}{*}{ Radiation dose (Gy) } & & & & & & & 10.446 & 0.005 \\
\hline & $<60$ & 47 & 40.0 & 10.3 & 6.9 & 9.0 & & \\
\hline & 60 & 186 & 57.5 & 27.7 & 22.9 & 14.0 & & \\
\hline & $>60$ & 111 & 52.2 & 19.1 & 11.4 & 12.3 & & \\
\hline
\end{tabular}


Figures

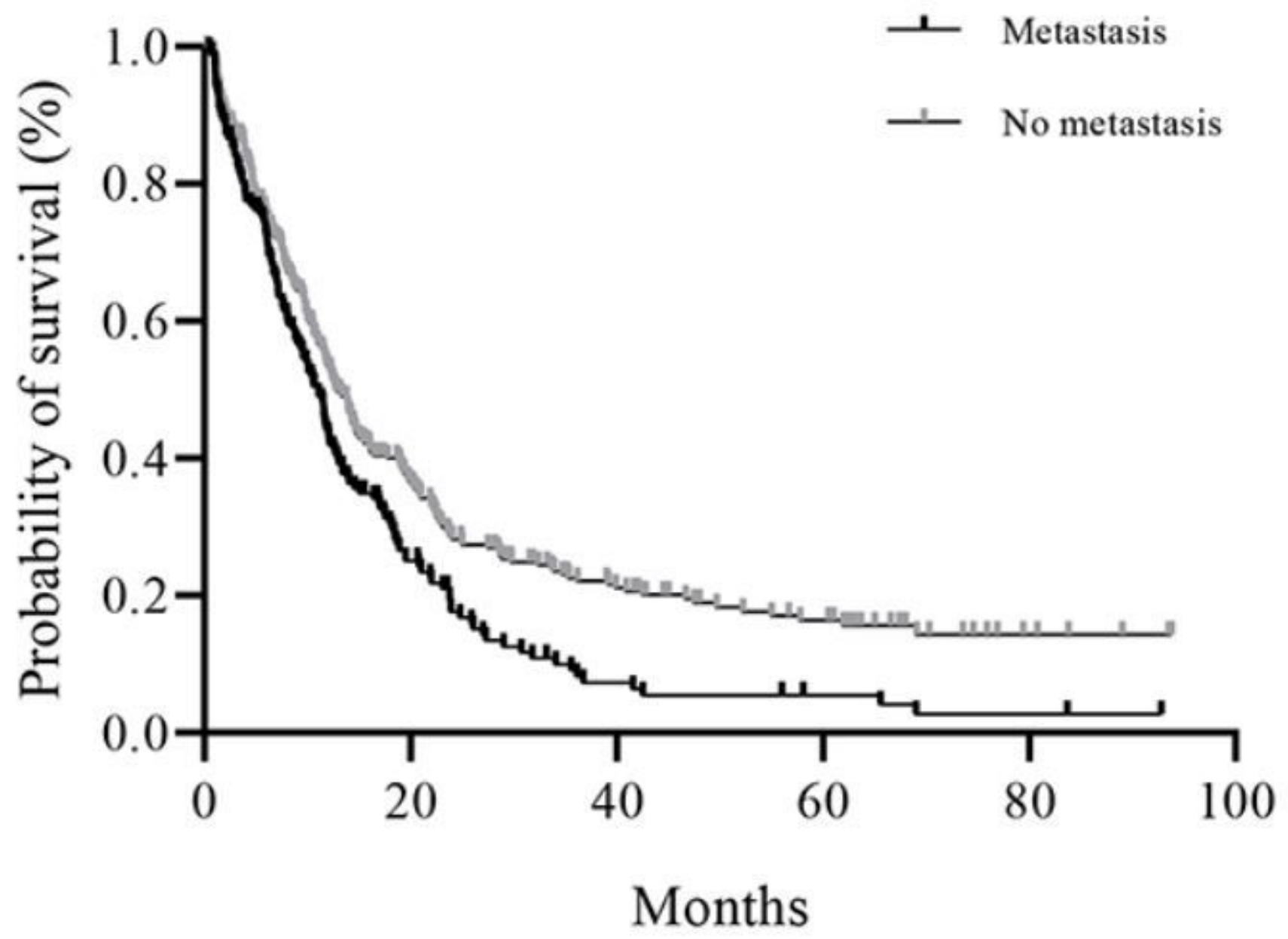

Figure 1

Survival curves of the cancer patients with or without combined combined distant metastasis. 

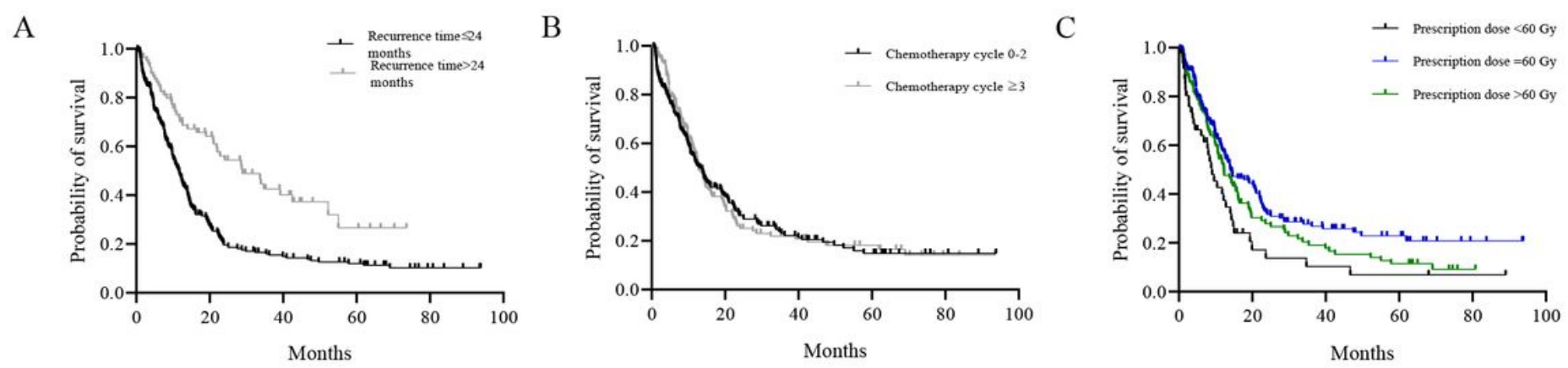

\section{Figure 2}

Single factor subgroup analysis curve of patients with postoperative local recurrence only. A: recurrence time, B: chemotherapy cycles, C: prescription dose. 